
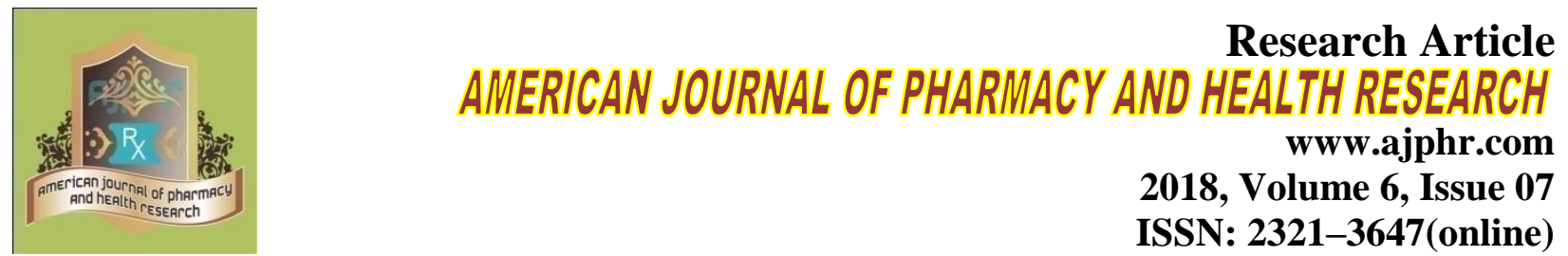

2018, Volume 6, Issue 07

ISSN: 2321-3647(online)

\title{
Design and Characterization of Biodegradable Chitosan Nanoparticles Loaded With Almotriptan Malate For Migraine Therapy
}

\author{
Akila RM ${ }^{1 *}$, Sneha Anna Saju ${ }^{1}$ \\ Department of Pharmaceutics, College of Pharmacy, Sri Ramakrishna Institute of Paramedical \\ Sciences, Coimbatore, India.
}

\begin{abstract}
Nanoparticles are designed to improve the pharmacological and therapeutic properties of conventional drugs. In the present research work, almotriptan malate was formulated as nanoparticle drug delivery system for the effective management of migraine by ion-gelation technique. Shape and size of the nanoparticles were evaluated by TEM images. FTIR and DSC studies confirmed that there were no interactions between the drug and the other ingredients. XRD study was carried out to confirm the crystalline or amorphous nature of the nanoparticles. The entrapment efficiency of was found to be 74 to $82 \%$ and drug loading capacity was found to be 15 to $16 \%$ The in-vitro release studies concluded that the release was sustained after an initial burst. The in vitro mucoadhesion study using goat nasal mucosa was found to be $54 \%$ and $69 \%$. The kinetic study revealed that the almotriptan malate nanoparticles followed the first order kinetics.
\end{abstract}

Keywords: biodegradable chitosan, nanoparticles, almotriptan malate, mucoadhesion 


\section{INTRODUCTION}

The method by which the drug is delivered can have a significant effect on its efficacy. Aiming therapeutic compound to the desired site is a major problem in treatment of many diseases. A traditional application of drugs is identified by limited action, poor bio- distribution and lack of acuteness[1]. These limitations and drawback can be overcome by controlling drug delivery. In controlled drug delivery system the drug is delivered to the place of action, thus its influence on important tissues and harmful side effect can be reduced. In addition controlled drug delivery system guards the drug from rapid, degradation or clearance and amplifies drug concentration in target tissues, therefore lower doses of drug required. Cell-specific targeting can be attained by attaching drugs to individually designed carriers. Chitosan nanoparticles have acquired more importance as drug delivery carriers because of their stability, low toxicity, simple and mild preparation method and yielding versatile routes of administration. Chitosan has the special possibility of adhering to the mucosal surfaces within the body because of their submicron size and is suitable for mucosal route i.e. oral, nasal and ocular mucosa which is a non-invasive route [2]. Diseases of the CNS such as schizophrenia, meningitis, migraine, Parkinsons disease and Alzheimers disease require delivery of the drug to the brain for treatment. However such conveyance remains difficult especially for hydrophilic and large molecular weight drugs due to the impervious nature of the endothelial membrane separating the systemic circulation and interstitial fluid of the blood brain barrier (BBB). Some of the currently employed invasive approaches include interstitial delivery, intracerebroventricular delivery, intracerebral delivery and nasal delivery[3]. A migraine is more than just a headache. It is a complicated neurological problem which can affect the whole body and can result in many symptoms, sometimes without a headache at all [4]. The triptan category of drugs is used in the termination of migraine attack by acting as vasoconstrictors by inhibiting neurogenic inflammation peripherally, inhibiting nociceptors[5]. As the oral route of delivery triptans has few disadvantages like nausea, the present research focuses on delivering almotriptan malate using chitosan nanoparticles as intranasal brain targeted delivery.

\section{MATERIALS AND METHOD}

Almotriptan malate was received as a gift sample from Azakem laboratories, Hyderabad, India. Chitosan ( $85 \%$ deacetylated), sodium tripolyphosphate were obtained from Sigma Aldrich, Mumbai and tween 80 was purchased from S.D Fine-Chem Pvt. Ltd. (Mumbai, India). All other reagents were of analytical grade and used as purchased. 


\section{Preparation of drug loaded chitosan nanoparticles [6]}

Chitosan nanoparticles were formulated based on the ionic gelation of chitosan and sodium tripolyphosphate anions. The weighed chitosan $(1,2,3 \mathrm{mg} / \mathrm{ml})$ was dissolved in $1 \% \mathrm{v} / \mathrm{v}$ of an aqueous solution of acetic acid, to which $0.5 \%$ of Tween 80 and almotriptan malate(2mg) was added. The sodium tripolyphosphate solution $(0.5,1,1.5 \mathrm{mg} / \mathrm{ml})$ was prepared in distilled water. This solution was added to the drug-loaded chitosan nanoparticles and stirred continuously using magnetic stirrer (REMI-2MLH) at room temperature for 30min. Subsequently, the pH was adjusted to 5.5 with the help of a required amount of $1 \mathrm{~N} \mathrm{HCl}$ or $\mathrm{NaOH}$ and then centrifuged at $10000 \mathrm{rpm}$ using a centrifuge for 30min, washed and dried.

Table 1 Formulation of Chitosan Nanoparticles

\begin{tabular}{llll}
\hline Batch code & $\begin{array}{l}\text { Chitosan } \\
(\mathbf{m g} / \mathbf{m l})\end{array}$ & $\begin{array}{l}\text { Sodium } \\
\text { tripolyphosphate(mg/ml) }\end{array}$ & $\begin{array}{l}\text { Almotriptan } \\
\text { malate(mg) }\end{array}$ \\
\hline B0 & 1 & 0.5 & 0 \\
B1 & 1 & 0.5 & 2 \\
B2 & 2 & 1 & 2 \\
B3 & 3 & 1.5 & 2 \\
\hline
\end{tabular}

\section{Characterization of nanoparticles}

\section{Invitro drug release study of the prepared formulations [7]}

The in vitro drug release study was performed in a USP Type II (Paddle type) apparatus using the principle of diffusion through the dialysis membrane. Drug-loaded nanoparticles redispersed in $2 \mathrm{ml}$ phosphate buffer ( $\mathrm{pH} \mathrm{6.5),} \mathrm{were} \mathrm{placed} \mathrm{in} \mathrm{a} \mathrm{dialysis} \mathrm{bag} \mathrm{soaked} \mathrm{in} \mathrm{distilled} \mathrm{water} \mathrm{1hour}$ prior to its use, and the bag was tied to the paddle of the apparatus. The paddle was rotated at 100 rpm and the dissolution media was $500 \mathrm{ml}$ of phosphate buffer $\mathrm{pH} 6.5$ maintained at $37 \pm 1{ }^{\circ} \mathrm{C}$. Sample aliquots of $5 \mathrm{ml}$ were collected at specific time intervals and were replenished with an equal amount of fresh dissolution medium. The samples were analyzed, spectrophotometrically at $\lambda \max =228 \mathrm{~nm}$ against suitable blank and amount of drug released at various time intervals were calculated. The results were expressed in terms of average $\%$ drug release \pm standard deviation.Invitro mucoadhesion studies [8]

\section{Invitro mucoadhesion studies [8]}

A piece of goat nasal mucosa was cleaned and placed in Krebs solution in Petri plate. It was washed with distilled water, and then accurately weighed almotriptan malate loaded chitosan nanoparticles equivalent to $100 \mathrm{mg}$ were spread on to it. The mucosa was kept aside for $15 \mathrm{~min}$. Then surface was washed with phosphate buffer $\mathrm{pH}$ 6.5. Nanoparticles retained on the mucosal surface after first washing was removed by rinsing thoroughly by phosphate buffer 6.5 . The 
solution was stirred to dissolve drug, filtered and absorbance was recorded. The amount of adhered nanoparticles was estimated as the difference between the amount of applied nanoparticles and the amount of flowed nanoparticles. The percent mucoadhesion was calculated using the following

$$
\text { percentage of mucoadhsesion }=\frac{\text { amount of drug in wash out liquid }}{\text { actual amount of drug in nanoparticles }} \times 100
$$

\section{In-vitro drug release kinetics study [9]}

In order to describe the mode of release of drug from nanoparticles the in-vitro release data of optimized formulation, B2 was fitted into the following mathematical models: (zero order, first order, Higuchi equation and Korsmeyer-Peppas equations).

$\mathrm{Qt}=\mathrm{K} 0 \mathrm{t}$ (Zero Order Kinetics)

$\log (\mathrm{Qt} / \mathrm{Q} 0)=-\mathrm{K} 1 \mathrm{t} / 2.303$ (First-order Kinetics)

$\mathrm{Qt}=\mathrm{K}_{\mathrm{KP}} \mathrm{t}_{\mathrm{n}}($ Korsmeyer and Peppas equation $)$

$\mathrm{Qt}=\mathrm{K}_{\mathrm{H}} \mathrm{t}^{1 / 2}$ (Higuchi's equation) Where, $\mathrm{Qt}$ is the percent of drug released at a time ' $\mathrm{t}$ ', $\mathrm{K}_{0}, \mathrm{~K}_{1}$, $\mathrm{K}_{\mathrm{KP}}$, and $\mathrm{K}_{\mathrm{H}}$ are the coefficients of Zero order, First order, Korsmeyer-Peppas and Higuchi's equations.

\section{Drug Content [10]}

$1 \mathrm{ml}$ of almotriptan malate loaded chitosan nanoparticles suspension is dissolved in the $10 \mathrm{ml}$ of distilled water. The amount of almotriptan malate was determined using UV spectrophotometer at $\lambda \max =228 \mathrm{~nm}$. The placebo formulation prepared similarly to drug-loaded chitosan is used as a blank. The total drug content was calculated.

$\mathrm{TDC}=$ concentration $\times$ dilution factor $\times$ volume of formulation $\times 100$

Transmission Electron Microscopy (TEM ) [11]

The nanoparticles of the optimized batch, after suitable dilution with HPLC grade water, were examined for surface morphology and particle size under a transmission electron microscope (Jeol/ JEM 2100 STIC, Cochin ), operated at $200 \mathrm{kV}$. The sample was placed on a carbon-coated copper grids and was stained with $2 \%$ phosphotungstic acid (freshly prepared, $\mathrm{pH}$ 7.0).

\section{Entrapment Efficiency and Drug Loading Capacity [12]}

The entrapment efficiency can be determined by measuring the concentration of the free drug in the dispersion. Entrapment efficiency and drug loading capacity of nanoparticles in different formulations are determined by ultra-centrifugation of samples at $10,000 \mathrm{rpm}$ for $30 \mathrm{~min}$. The amount of free almotriptan malate was determined in the clear supernatant by UV spectrophotometry at $227 \mathrm{~nm}$ using supernatant of non-loaded (blank) nanoparticles as the basic 
correction. The drug loading capacity of nanoparticles and entrapment efficiency (EE) of the process were calculated from the following equations:

$$
\begin{gathered}
\text { Entrapment Efficiency }(E E \%)=\frac{\text { total amount of drug }- \text { free drug }}{\text { total drug }} \times 100 \\
\text { Drug loading capacity }=\frac{\text { total amount of drug }- \text { free drug }}{\text { total weight of nanoparticles }} \times 100
\end{gathered}
$$

\section{Infrared (IR) spectrum[13]}

The IR spectra of the formulations were carried out for drug-loaded chitosan nanoparticles as well as for the physical mixture of drug and excipients using the JASCO FTIR 4100 instrument in the frequency range of $400-4000 \mathrm{~cm}^{-1}$ with the resolution of $4 \mathrm{~cm}^{-1}$

\section{Differential scanning calorimetry (DSC) [14]}

DSC studies were performed to understand the behavior of cross-linked chitosan on the application of thermal energy. DSC was performed on a DSC-7 (Perkin-Elmer Corp., USA) at a heating rate of $0^{\circ} \mathrm{C} / \mathrm{min}$ in the temperature range of $0-350^{\circ} \mathrm{C}$ using the empty aluminum pan as a reference standard

\section{Powder X-ray diffraction study [15]}

The XRD patterns of pure drug, dummy nanoparticle and optimized nanoparticle are to be measured on a diffractometer. Monochromatic radiation $\left(\lambda=1.5418 \mathrm{~A}^{\circ}\right)$, at $40 \mathrm{kV}$ and $40 \mathrm{~mA}$, was used as the X-ray source. The diffractograms were recorded between $20^{\circ}$ and $70^{\circ}$ with an increasing step of $0.02^{\circ}$ and $2 \mathrm{~s}$ as the time for each step.

\section{Stability studies[16]}

The stability study was carried out to assess the stability of almotriptan malate loaded chitosan nanoparticles. For this purpose, samples were kept in borosilicate glass vials and stored at room temperature, in a refrigerator $\left(5 \pm 1^{\circ} \mathrm{C}\right)$, and $45 \pm 1{ }^{\circ} \mathrm{C}(75 \%$ relative humidity $)$ in the stability chamber. Samples were analyzed at the intervals of $0,7,14,21,28,35$, and 45 days for their changes in physical appearance and for the drug content.

\section{RESULTS AND DISCUSSION}

\section{In-vitro drug release study}

The in-vitro release profile of almotriptan malate from different formulations is shown in Figure1. The percent drug release was not same for all the formulations, it varies with time.

The release profile for chitosan biodegradable nanoparticles was obtained in phosphate buffer solution, $\mathrm{pH}$ 6.5. Initially, the chitosan nanoparticles showed the immediate release of almotriptan malate, with about $45 \%$ almotriptan malate being released in 10 minutes which may 
be due to release of drug from the surface nanoparticles, followed by slow release of drug which is encapsulated in chitosan nanoparticles. When the particle size of drug loaded chitosan, nanoparticles was smaller, the surface area tends to become larger and hence the drug release was faster and when the concentration of chitosan was less, the drug release was high (B1>B2>>B3).

Table 2: Cumulative percentage drug release studies

\begin{tabular}{cccc}
\hline \multirow{2}{*}{ Time $(\mathbf{m i n})$} & \multicolumn{3}{c}{ Cumulative percentage drug release } \\
\cline { 2 - 4 } & Batch1 & Batch 2 & Batch 3 \\
\hline 0 & 0 & 0 & 0 \\
5 & 37 & 32 & 28 \\
10 & 45 & 43 & 39 \\
15 & 54 & 51 & 48 \\
30 & 57 & 53 & 50 \\
45 & 66 & 60 & 58 \\
60 & 71 & 65 & 61 \\
75 & 75 & 71 & 66 \\
90 & 79 & 75 & 72 \\
\hline
\end{tabular}

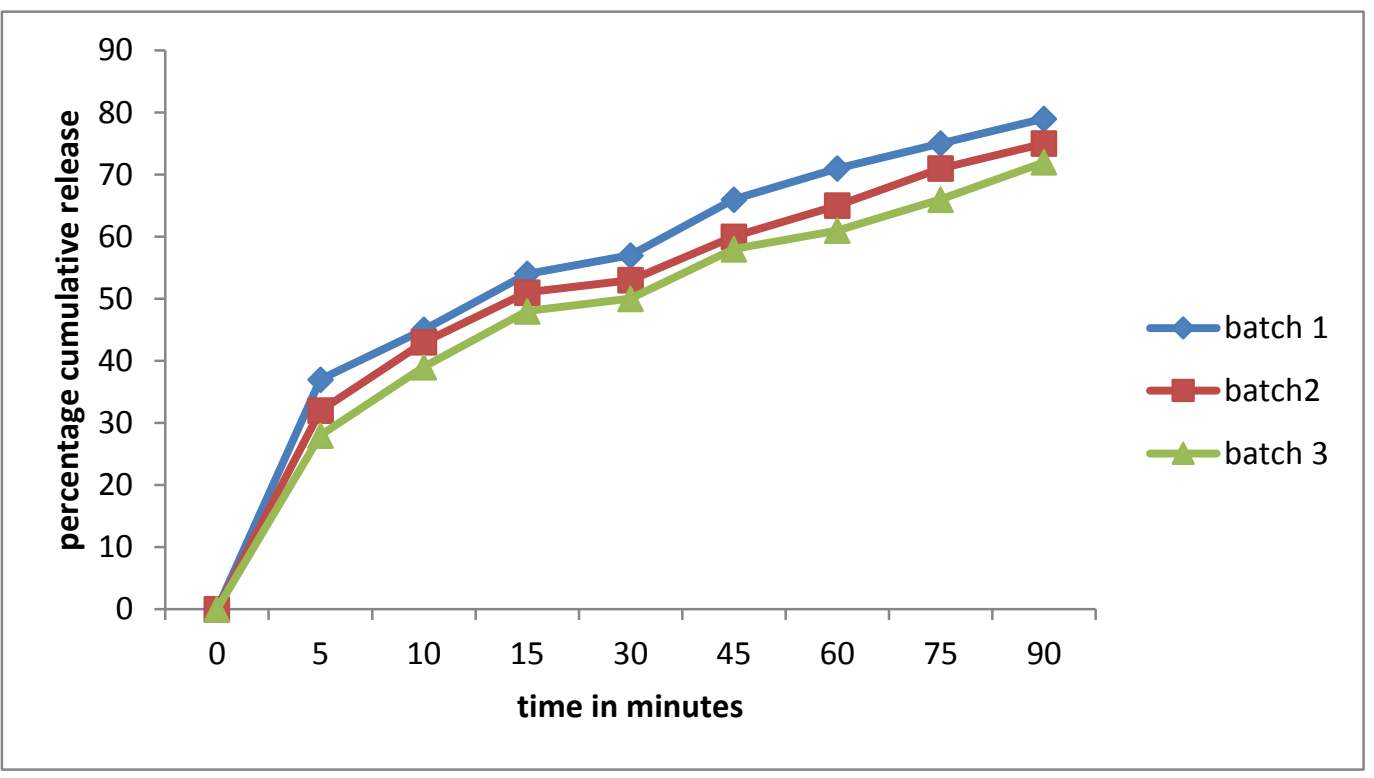

Figure 1: In vitro drug release studies

\section{Mucoadhesion Test}

Mucoadhesion test was performed to ensure the adhesion of formulation to the nasal mucosa for a prolllonged period at the site of absorption. The results of the mucoadhesion studies are shown in the table3. The results indicated that amount of chitosan and volume of sodium tripolyphosphate was directly proportional to mucoadhesion strength. This could be attributed to the availability of a high amount of polymer for interaction with mucus. 
Table 3: Percentage Mucoadhesion Of The Drug Loaded Nanoparticles

\begin{tabular}{lll}
\hline Sl. No & Batch code & $\begin{array}{l}\text { Percentage mucoadhesion }(\%) \\
\text { Of the drug loaded Nanoparticles }\end{array}$ \\
\hline 1 & $\mathrm{~B}_{1}$ & 69 \\
2 & $\mathrm{~B}_{2}$ & 61 \\
3 & $\mathrm{~B}_{3}$ & 54 \\
\hline
\end{tabular}

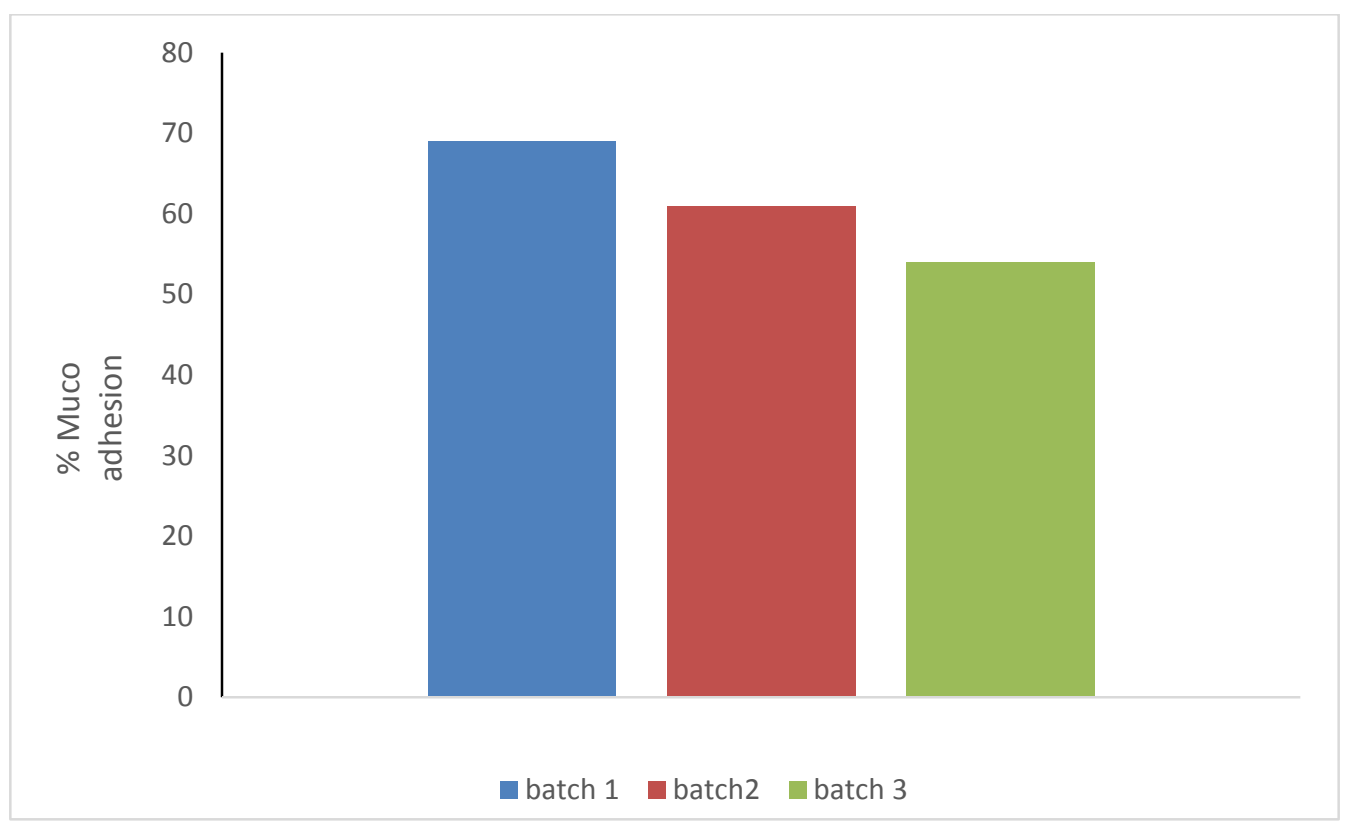

Figure 2: Percentage Mucoadhesion Of The Drug Loaded Nanoparticles

\section{In-vitro drug release kinetic study}

To analyze the mechanism for the release and release rate kinetics of the dosage form, the data obtained from in-vitro drug release from optimized formulation (B1)was fitted to model's representation zero order, first order, Higuchi, and Korsmeyer-Peppas. The release data, when fitted into release kinetic equations, produced correlation coefficients (R2) of 0.917, 0.916 and 0.905 for the first order, Korsmeyer-Peppas, and Higuchi models respectively and non-linearity when plotted by the zero-order equation (Figure 3-6). Hence, it can be concluded that the major mechanism of drug release follows first order kinetics. Data based on the first order models usually provide evidence to the diffusion mechanism of drug release from sustained release delivery systems.

\section{Drug content}

The drug content ranges from $82 \%$ to $91 \%$ and it is more in B3 as it was trapped in a higher concentration of chitosan.

\section{Entrapment Efficiency}


The drug entrapment efficiency was found to be in a range of $73.5 \%$ to $81.9 \%$ and this indicates that when chitosan concentration is more the drug is trapped in the chitosan network and are diffused slowly into the environment. (Figure 4)

\section{Loading capacity}

The loading capacity decreases from $16.38 \%$ to $14.79 \%$. and this indicates when chitosan concentration is less, drug is loaded more (Figure 4)

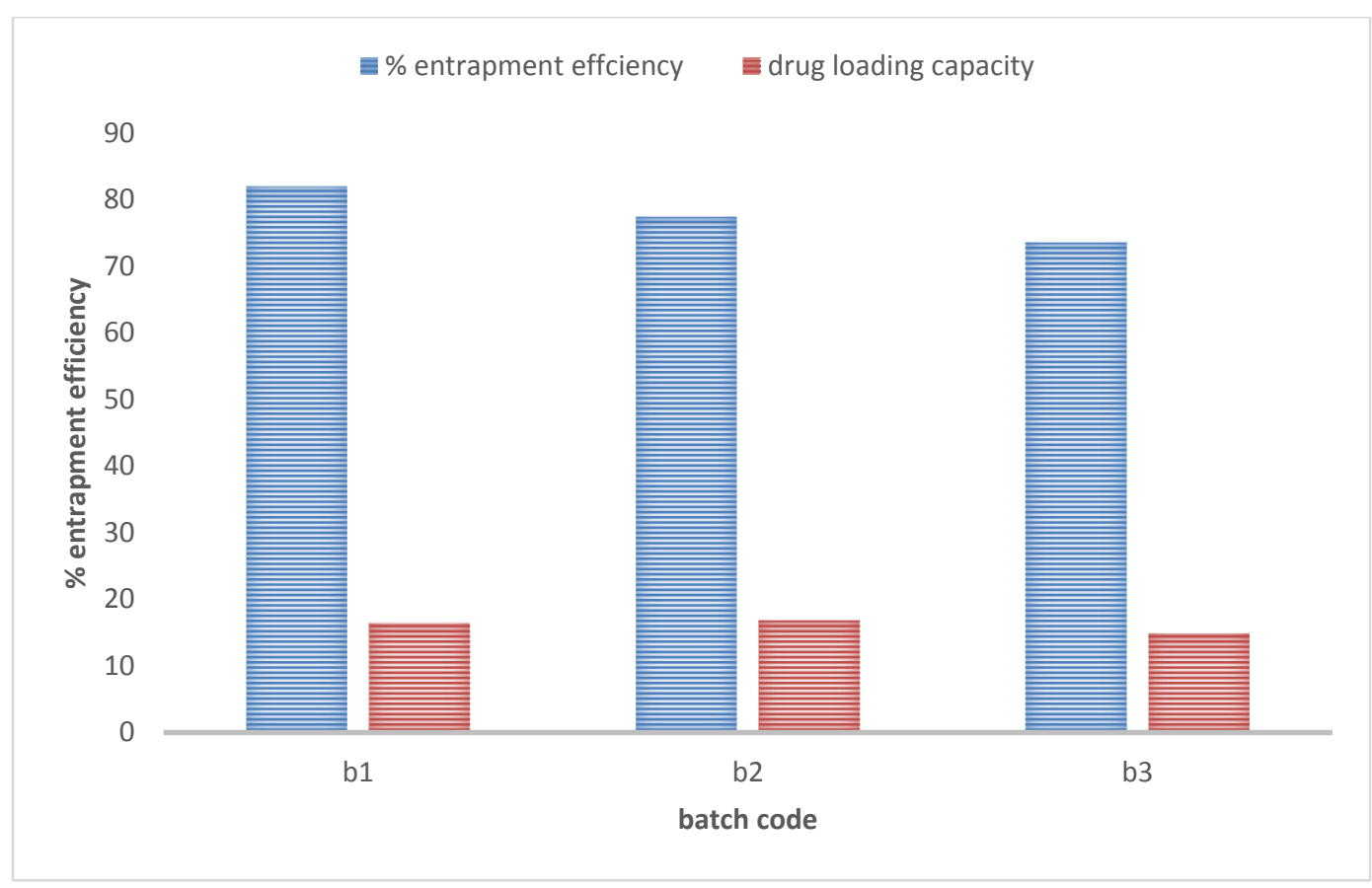

Figure 3: Entrapment Efficiency and Drug Loading Capacity

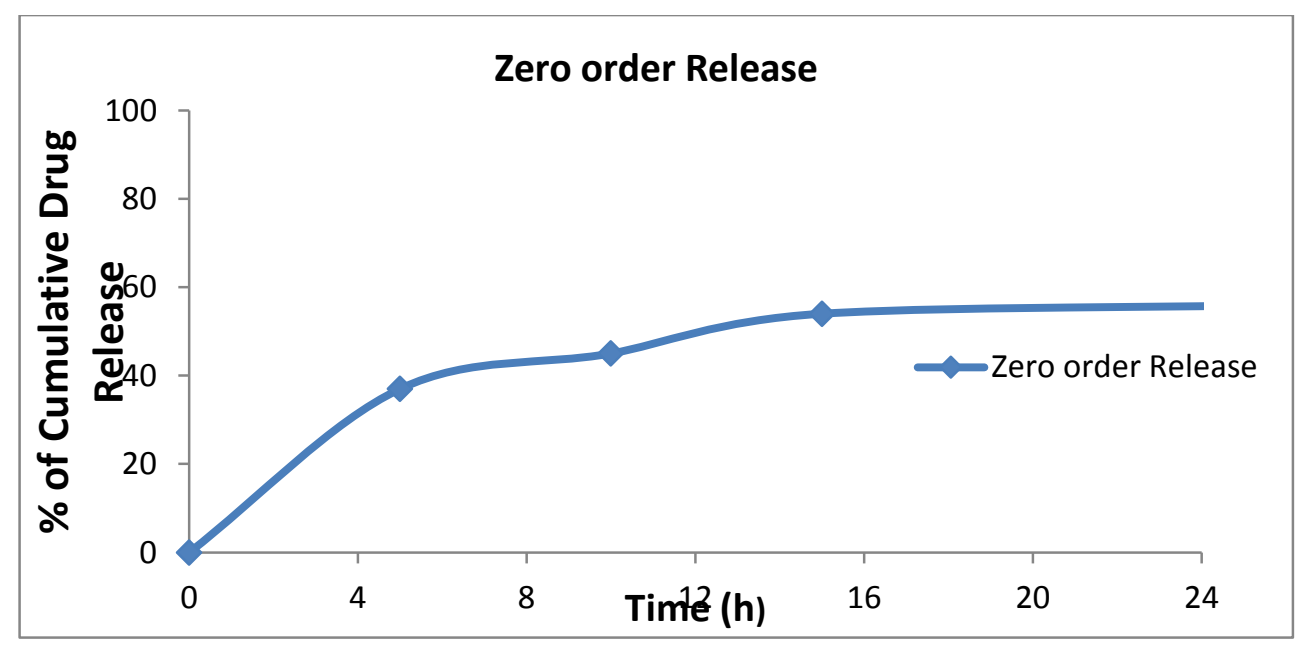

Figure 4 


\section{Akila et al.,}

Am. J. Pharm Health Res 2018;6(07)

ISSN: 2321-3647

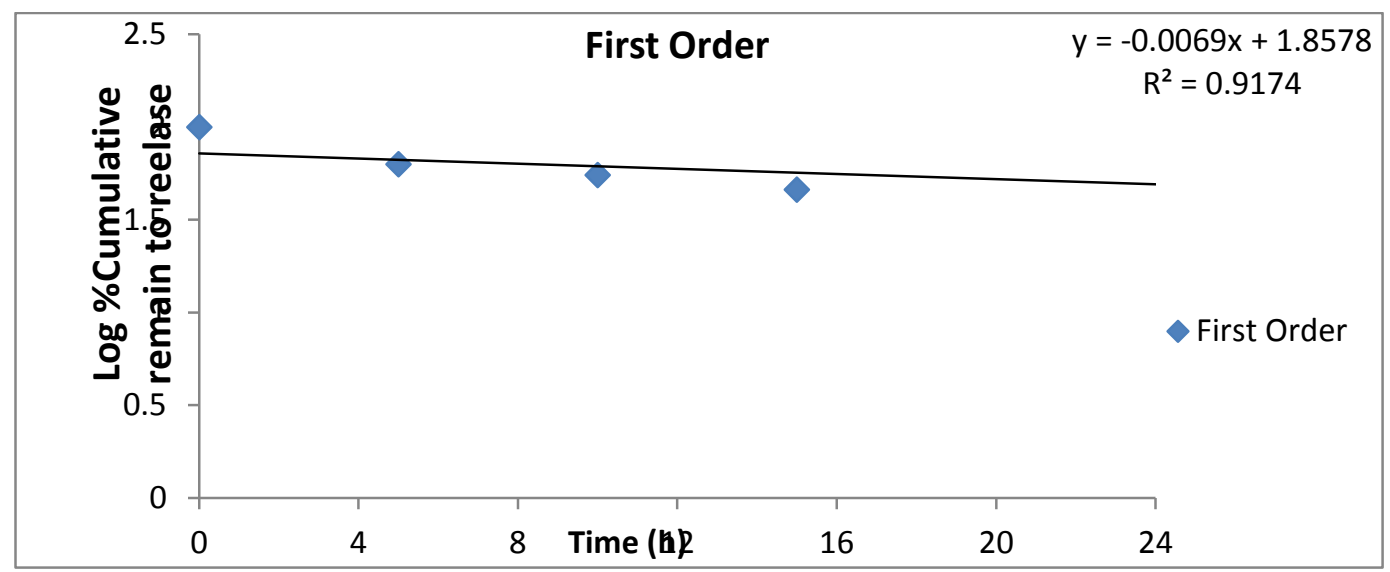

Figure 5

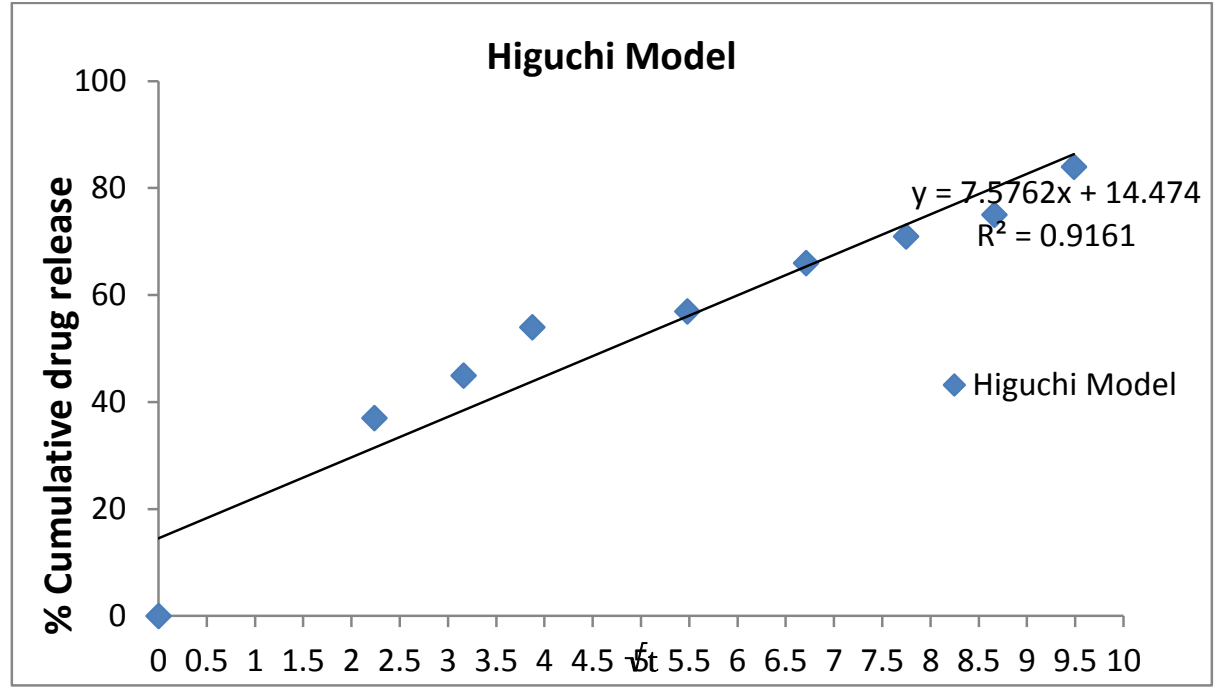

Figure 6

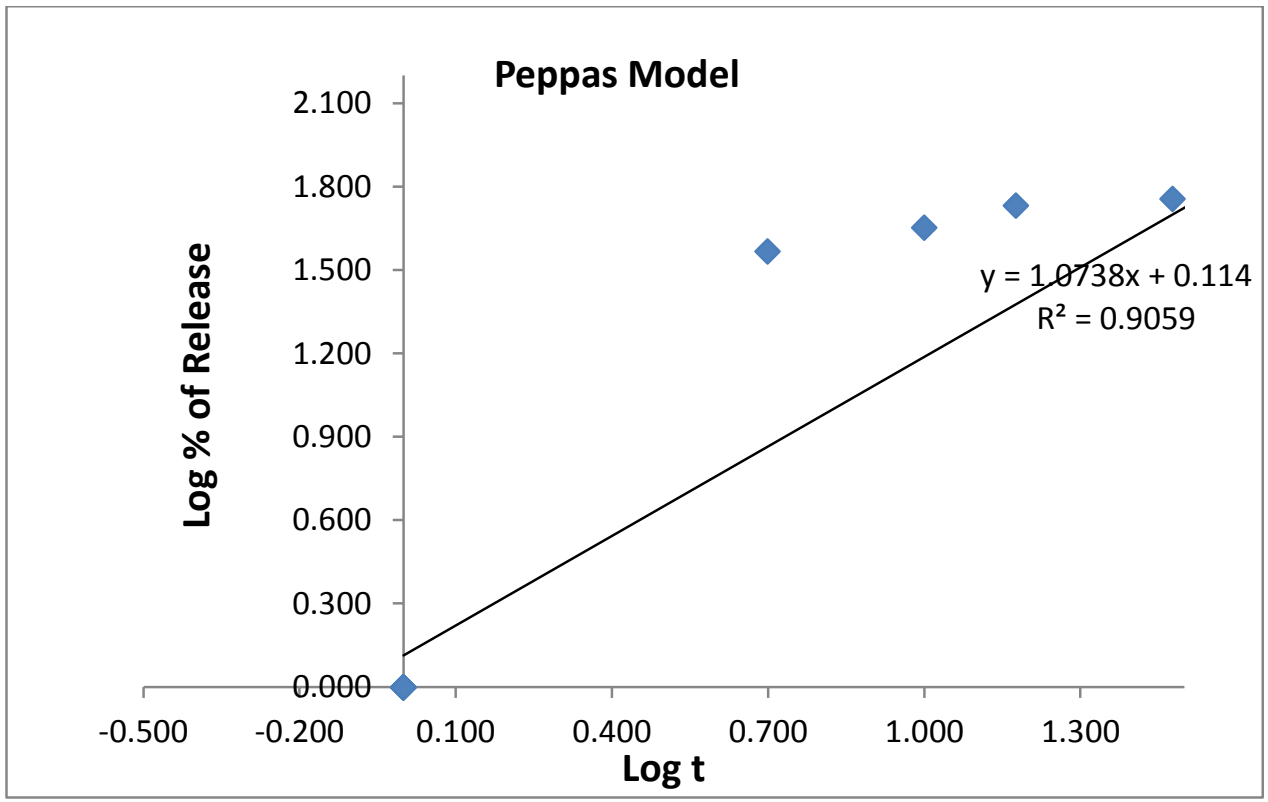

Figure 7 


\section{Infrared IR spectrum}

The FT-IR spectrum corresponding to the drug- loaded chitosan nanoparticles and physical mixture of drug and excipients confirmed the absence of any chemical interaction between the drug and carriers because their individual characteristic peaks were obtained in the spectra of the formulation. Also, it confirmed that almotriptan malate incorporated in nanoparticles retained its salt form.(Figure 8-9)

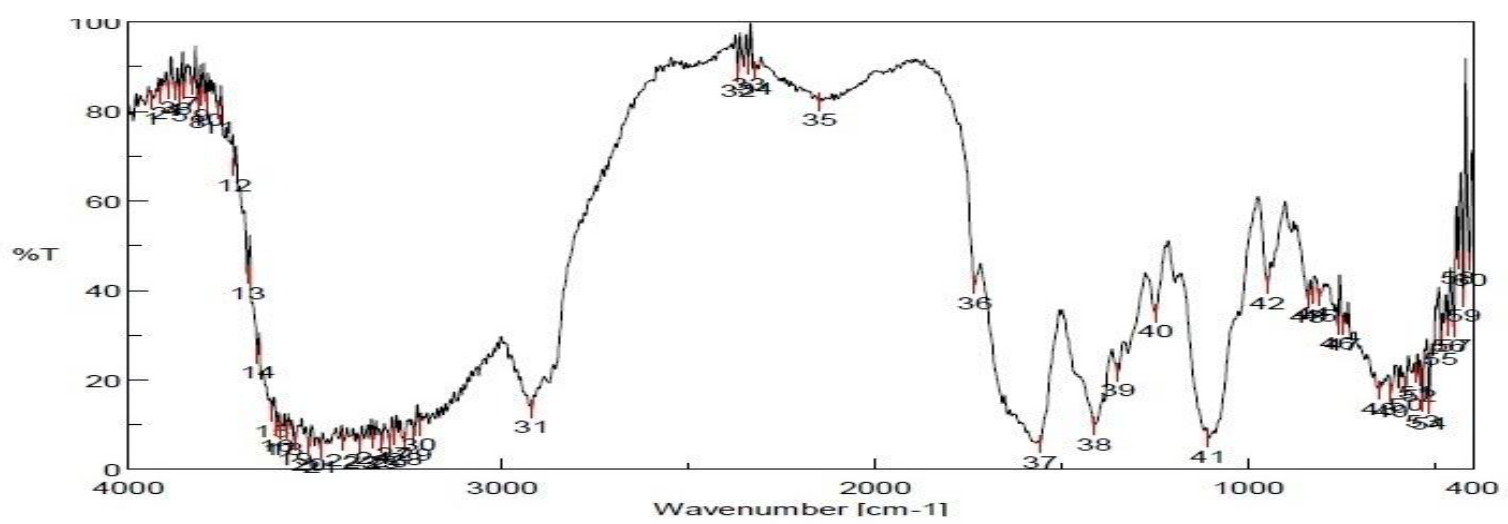

Figure 8: IR spectrum of drug- loaded chitosan nanoparticles

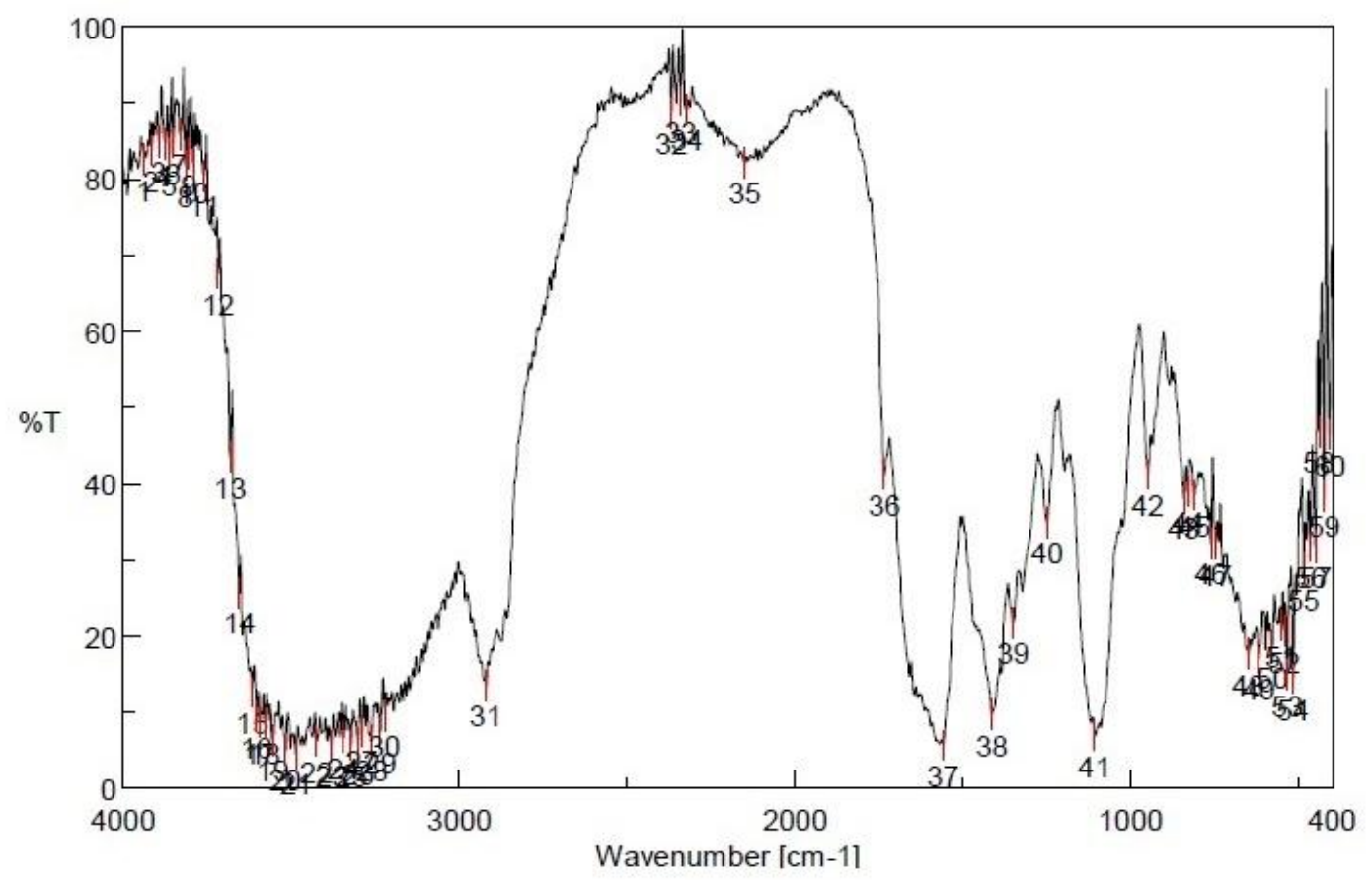

Figure 9: IR spectrum of physical mixture of drug and excipients

\section{Morphological characterization and particle size determination of nanoparticles}

Particle morphology and particle size were examined by transmission electron microscopy (TEM). The TEM image of drug loaded optimized chitosan nanoparticle, reveals that the particles were segregated, uniform in size, spherical in shape(Figure 10-13). The nanoparticles 
were within a narrow size distribution range in B0, B1 and B2 batches and particle size slightly increased in B3 batch with the high concentration of each composition (Table 4).

Table 4: Particle size determination

\begin{tabular}{lll}
\hline Sl. No & Batch code & Size range $(\mathbf{n m})$ \\
\hline 1 & $\mathrm{~B}_{0}$ & $100 \mathrm{~nm}$ \\
2 & $\mathrm{~B}_{1}$ & $100 \mathrm{~nm}$ \\
3 & $\mathrm{~B}_{2}$ & $159 \mathrm{~nm}$ \\
4 & $\mathrm{~B}_{3}$ & $183 \mathrm{~nm}$ \\
\hline & & \\
& &
\end{tabular}

Figure 10

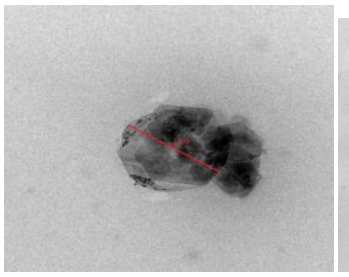

Figure12
Figure 11

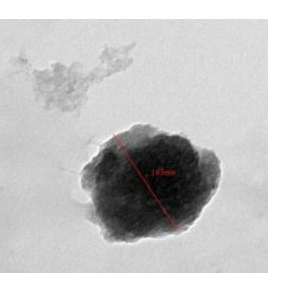

Figure 13

\section{Differential scanning calorimetry(DSC)}

The DSC thermograms reveal the melting point and the crystalline or amorphous behavior of the drug and the carriers(Figure 14- 16).

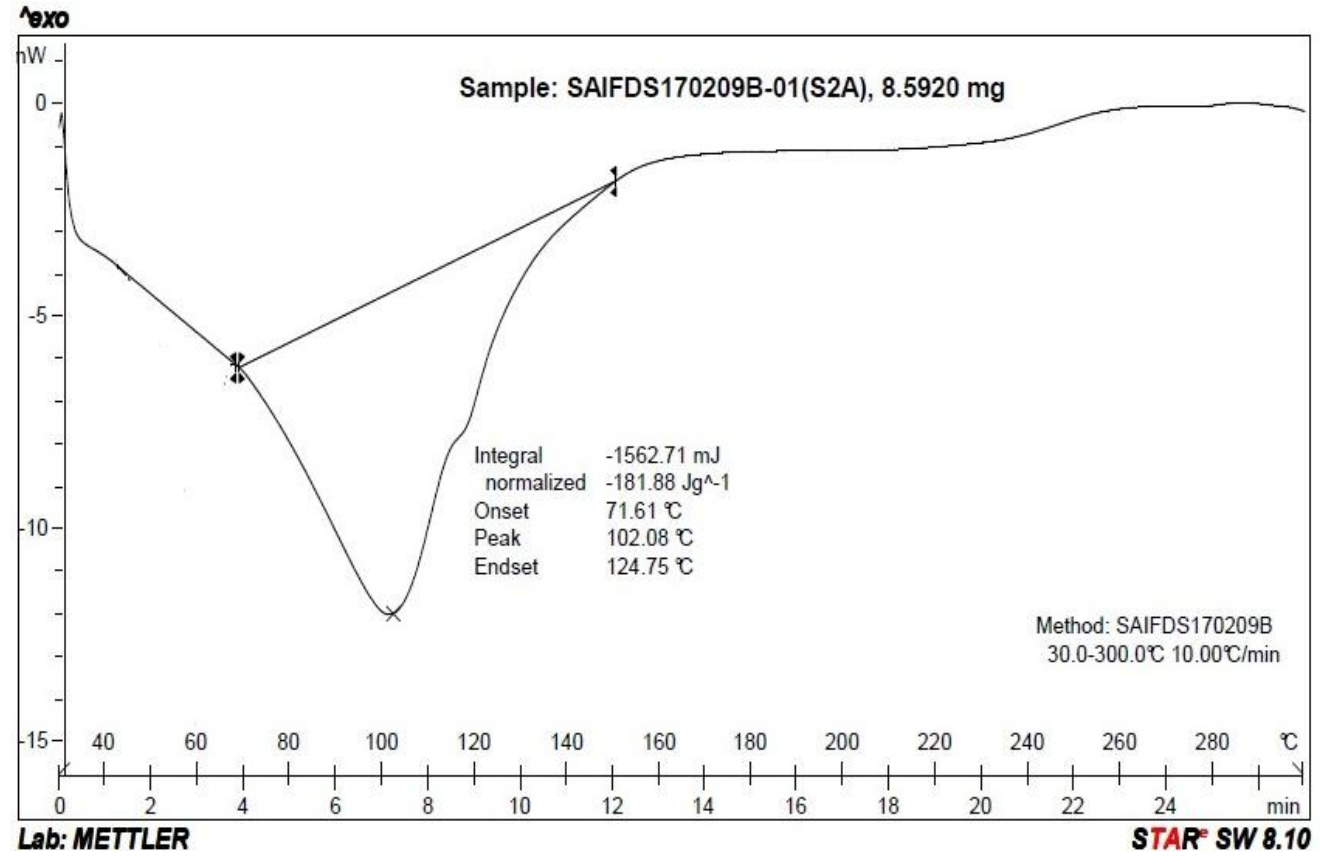

Figure 14: DSC Thermogram Of Drug Almotriptan malate 
Thermal analysis indicated that the DSC scan of the drug presented a sharp endothermic peak. A sharp endothermic peak was obtained at $124.75^{\circ} \mathrm{C}$ in the thermogram of the pure drug almotriptan malate, indicating its melting point temperature and crystalline state.

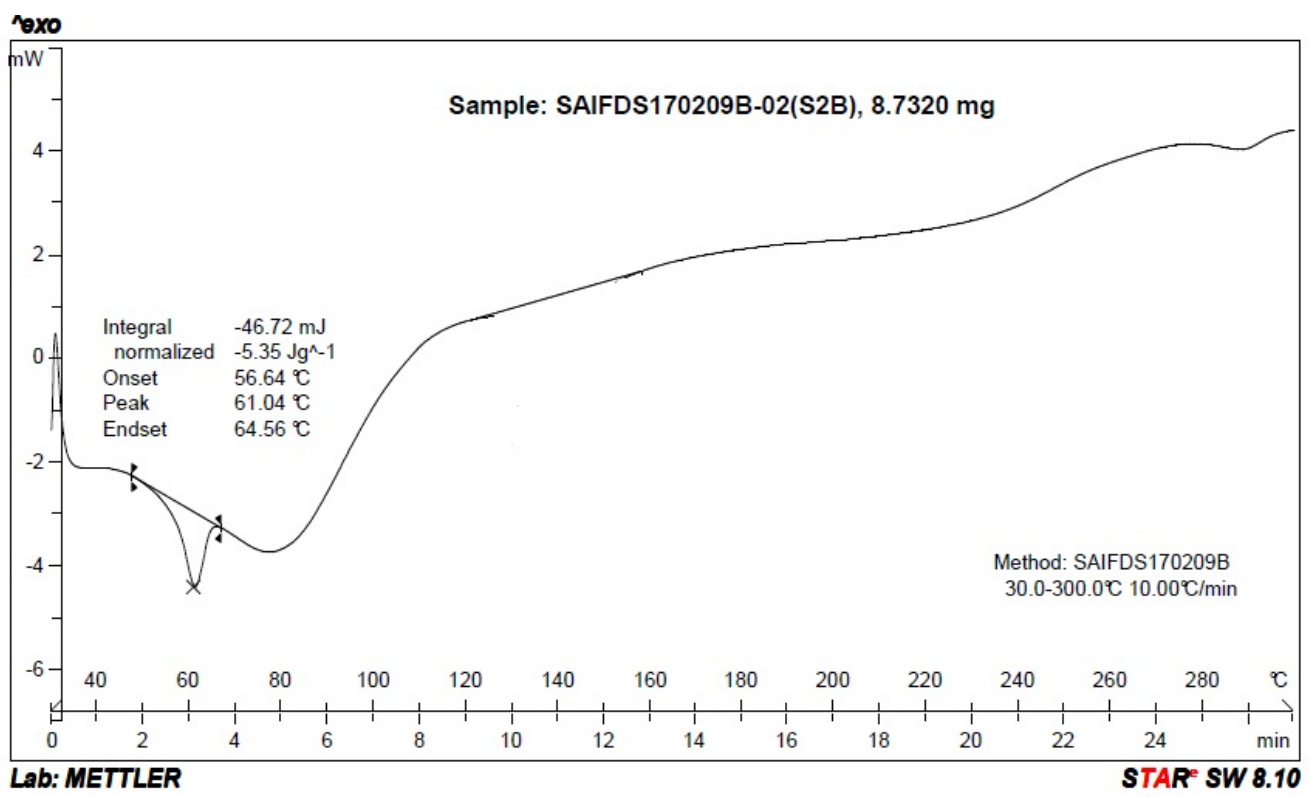

Figure 15: DSC Thermogram Of Almotriptan malateotriptan Loaded Nanoparticles

The endothermic peaks at $64^{\circ} \mathrm{C}$ in the thermogram of blank nanoparticles corresponded to the melting point of sodium tripolyphosphate, present in the formulation. The melting point peaks of chitosan were not observed because of their amorphous nature.

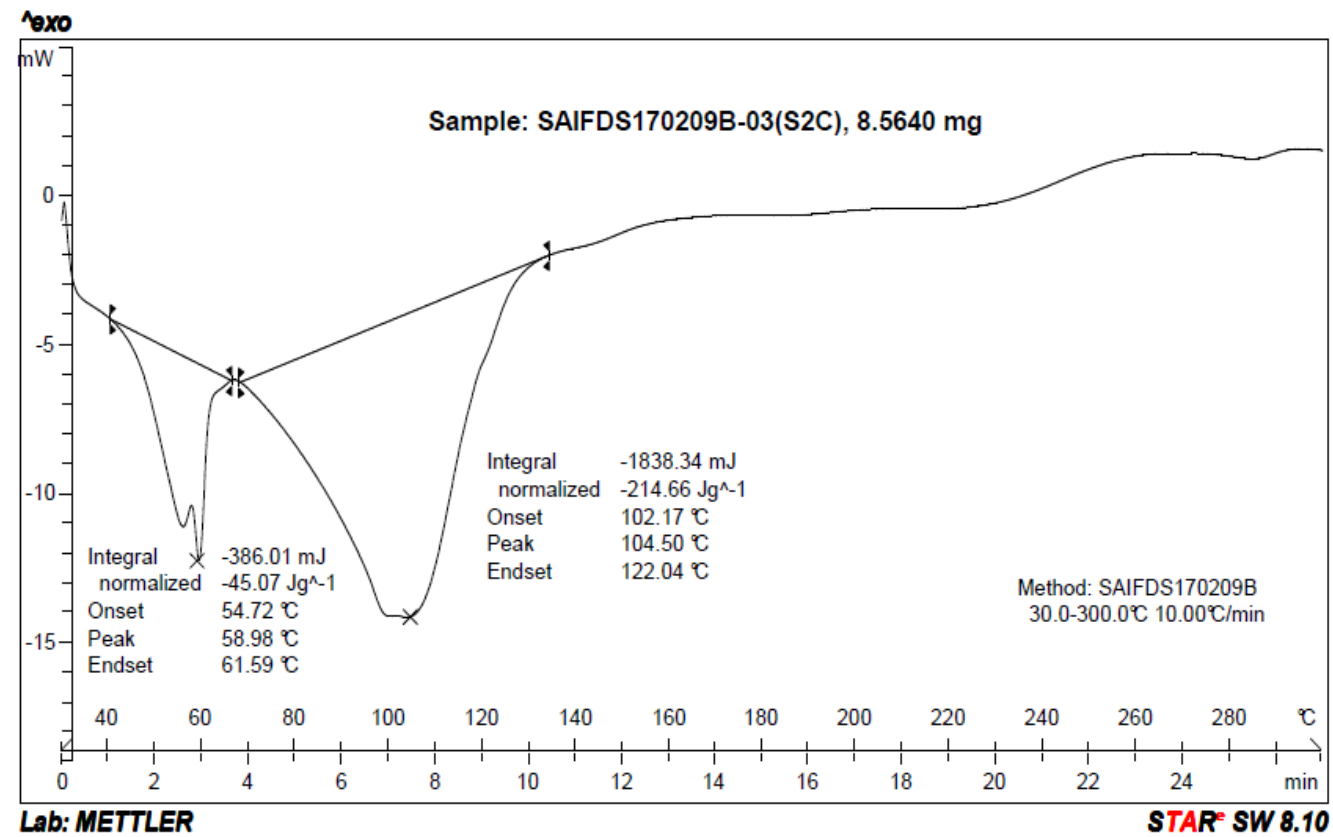

Figure 16: DSC Thermogram Of Blank Nanoparticles

DSC spectrum of Chitosan Nanoparticles loaded with drug almotriptan malate does not exhibit 
the sharp peak of almotriptan malate. Thus, we can have concluded that in the chitosan nanoparticles of almotriptan malate, the drug was in the partially crystalline state in chitosan nanoparticles and there is no interaction between drug and polymer.

\section{Powder X-ray diffraction study}

XRD studies are used to investigate the crystallinity of drug in the chitosan nanoparticles. The Xray diffractograms recorded for pure almotriptan malate, blank nanoparticles, and drug-loaded nanoparticles are presented. almotriptan malate revealed characteristic intense peaks at $2 \theta$ of $16^{\circ}$, $17^{\circ}$ and $22^{\circ}$ which are due to crystalline nature of almotriptan malate. However, in case of blank nanoparticles and drug-loaded chitosan nanoparticles, no intense peaks were observed between $2 \theta$ of $16^{\circ}, 17^{\circ}$ and $22^{\circ}$ indicating amorphous nature of the drug substance after entrapment into chitosan nanoparticles. It can be concluded that drug particles are dispersed at the molecular level in the polymer matrices since no indication about the crystalline nature of the drug was observed in the drug-loaded chitosan nanoparticles (Figure 17).

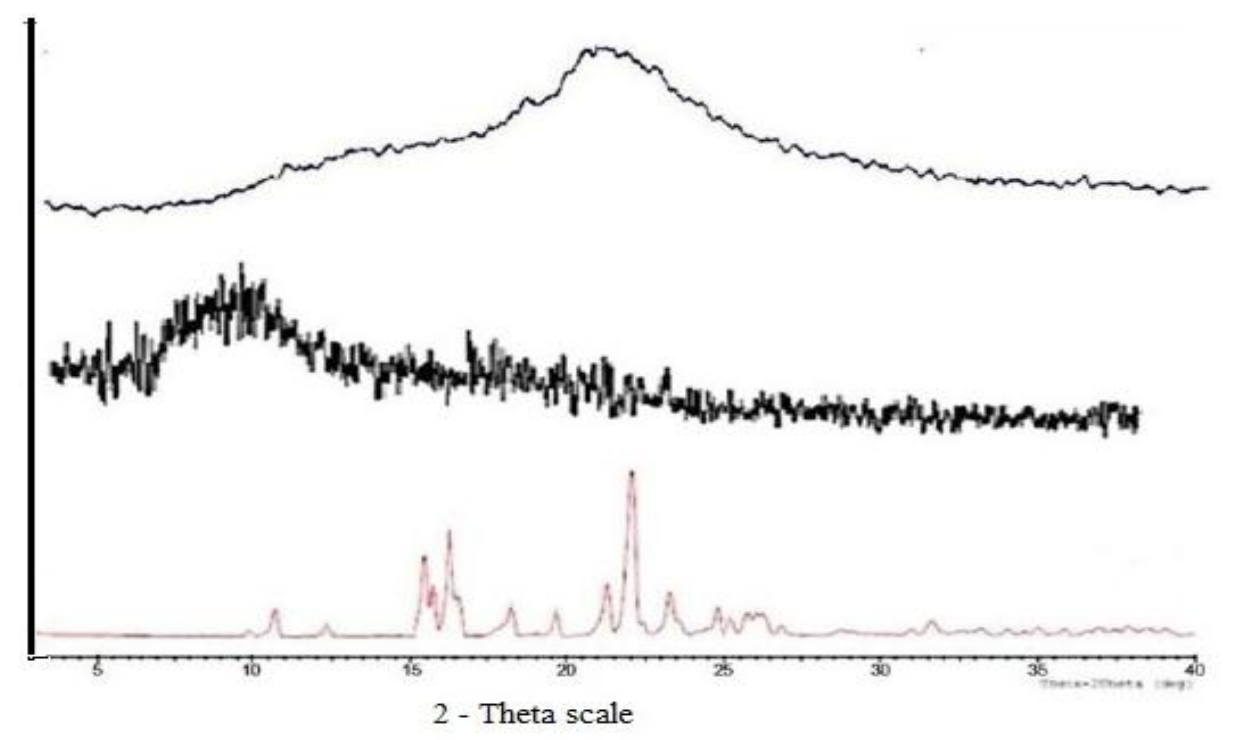

Figure 17: Powder Diffraction Studies Of Drug, Drug Loaded Nanoparticles, Blank Nanoparticles

\section{Stability studies}

The nanoparticles were taken and observed their physical properties at an interval of 7 , $14,21,28,35$ and 45 days. no change was observed in their physical appearance. The colour remains the same. The drug content remains unchanged at $5 \pm 1{ }^{\circ} \mathrm{C} \&$ room temperature Table 5 . 
Table 5: Drug Content in Stability Studies

\begin{tabular}{|c|c|c|c|c|}
\hline \multirow[t]{2}{*}{ Sl.no } & \multirow{2}{*}{$\begin{array}{l}\text { Sampling } \\
\text { interval days }\end{array}$} & \multicolumn{3}{|c|}{ Drug content } \\
\hline & & $5 \pm 1^{\circ} \mathrm{C}$ & Room temperature & $45 \pm 2^{\circ} \mathrm{C}$ \\
\hline 1 & 7 & 100 & 100 & 100 \\
\hline 2 & 14 & 99.5 & 99.5 & 99.5 \\
\hline 3 & 21 & 99.2 & 98.4 & 98.3 \\
\hline 4 & 28 & 99.1 & 97.4 & 86.5 \\
\hline 5 & 35 & 98.8 & 96.5 & 77.9 \\
\hline 6 & 45 & 98.1 & 94.3 & 74.3 \\
\hline
\end{tabular}

\section{CONCLUSION}

Almotriptan malate loaded chitosan nanoparticles were successfully formulated via the ionotropic gelation technique. The obtained nanoparticles easily penetrate the nasal mucosa by virtue of particle size. The present investigation, hence, led to successful nose to brain targeting of the hydrophilic drug almotriptan malate by its incorporation in chitosan biodegradable nanoparticles for the efficient therapeutic management of migraine. The validity of this work may be confirmed by in-vivo studies and the free drug could be combined with the penetration enhancers to enhance the release of the drug to provide quick action and prolonged release to treat mild to moderate migraine. All results of this research work provide useful information for future studies aiming at development of drug delivery formulation consisting of chitosan nanoparticles and with drug almotriptan malate for treatment of migraine.

\section{ACKNOWLEDGEMENTS}

The authors sincerely thank Dr. M. Gopal Rao, Professor \& Head, Department of Pharmaceutics, College of Pharmacy, Sri Ramakrishna Hospital Campus, Coimbatore for providing necessary facilities to carry out this work.

\section{REFERENCES}

1. Bhagwat RR, Vaidhya, IS. Novel Drug Delivery Systems: An Overview. Int J Pharm Sci Res. 2013; 4:970-82.

2. Ahmad RA,Ferky A. Preparation and characterization of nanoparticles of modified chitosan sensor and its application for the determination of heavy metals from different aqueous media. Int J Electrochem Sci. 2013; 8:6692-6708.

3. Chatterjee B. Nose to Brain drug delivery: A Recent Update. J formul Sci Bioavailab. 2017; 1:100-105. 
4. Noseda R, Burstein R. Migraine pathophysiology: Anatomy of the trigeminovascular pathway and associated neurological symptoms, CSD, sensitisation and modulation of pain. Pain 2013;154:S44-S53.

5. Andrew HA, Allan IB. Where do triptans act in the treatment of migraine? Pain 2005; 115:1-4.

6. Satish KM, Saugat Adhikari, Nisharani SR.Formulation and invitro characterization of chitosan biodegradable nanoparticles of zolmitriptan for migraine treatment. Pharmacie Globale Int J Comprehensive Pharmacy 2013;4: 1-5.

7. Sagar SJ, Aparna V.B. Formulation and characterization of chitosan nanoparticles loaded with rizatriptan benzoate. Der Pharmacia Lettre. 2013; $5: 218-223$

8. Khom TC, Hemant KSY, Abhay R, Navya M, Hemant SK, Sankeerth NK.Development of mucoadhesive nanoparticulate system of ebastine for nasal drug delivery.Trop J Pharm Res .2014; 13:1013-1019.

9. Balaiah A, Ephraiem Babu G, Vijayalakshmi P, Naga Raju K, Deepika B.Formulation development and invitro characterization of oral levetiracetam microspheres.International Res J Pharm. App Sci.2012; 2:13-21.

10. Satish PK, Kalpesh WS, Kamlesh M, Dheeraj B, Dinesh JK. Development And Evaluation Of Solid Lipid Nanoparticles Containing Anti-Migraine Drug, World J Pharm Sci. 2014.; 2:1014-1021.

11. Hansraj GP, Singh SK, Kumar P, Sumatriptan succinate loaded chitosan solid lipid nanoparticles for enhanced anti-migraine potential. Int J Biol Macromol. 2015; 5: 467477.

12. Farshid A, Lakshmi CSR. Formulation of nanoparticles of antimigraine drugs triptans by coacervation method. Asian J Pharm Clin Res. .2017; 10: 122-128.

13. Riddhe Dave, Rakesh Patel. Preparation and characterization of doxorubicin Hcl loaded chitosan nanoparticles by w/o emulsion method. Int J Pharm Life Sci.2013; 4:2407-2413.

14. Suresh G, Manjunath K, Venkateswarlu V, Satyanarayana V.Preparation ,characterization and in vitro evaluation of lovastatin solid lipid nanoparticles..AAPS PharmSciTech. 2013;8: E162-170.

15. Pooja TS, Sunil KY, Pallavi SP.Development and characterization of chitosan nanoparticles containing erythromycin estolate.International journal of pharmaceutical applications.2014; 1:1-7. 
16. Neha G, Upendra N, Shubhini AS, Intranasal delivery of chitosan nanoparticles for migraine therapy. Sci Pharm.2013;3:843-854.

AJPHR is

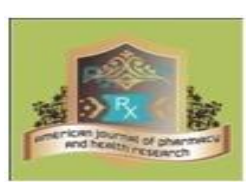

Peer-reviewed

monthly

Rapid publication

Submit your next manuscript at

editor@ajphr.com / editor.ajphr@gmail.com 